\title{
Location of Death
}

National Cancer Institute

\section{Source}

National Cancer Institute. Location of Death. NCI Thesaurus. Code C124331.

The physical place at which the subject ceased to live. 